\title{
Erratum: Small-world networks: Evidence for a crossover picture [Phys. Rev. Lett. 82, 3180 (1999)]
}

\author{
Marc Barthélémy and Luís A. Nunes Amaral
}

We have performed new calculations using the breadth-first search algorithm $[1,2]$. We are now able to study systems with sizes up to $n=5500$. As shown in Fig. 1, we now find $\tau \approx 1$, in agreement with the simple argument given in our Letter but different from the originally reported numerical result $(\tau=0.67 \pm 0.10)$. The reason for the incorrect numerical result reported initially is the small system sizes we studied, which did not allow us to reach the asymptotic regime.

We thank M.E.J. Newman and D.J. Watts [3] and A. Barrat [4] for alerting us to the possibility of an error on our estimate of $\tau$. We also thank M. Argollo de Menezes for directing us to the breadth-first search algorithm.

[1] J. van Leeuwen, Ed., Handbook of Theoretical Computer Science. Volume A: Algorithms and Complexity (Elsevier, Amsterdam, 1990), p. 539.

[2] We found the LEDA libraries very useful and efficient [http://www.mpi5b.mpg.de/LEDA/leda.htm].

[3] M.E.J. Newman and D.J. Watts, cond-mat/9903357.

[4] A. Barrat, cond-mat/9903323; A. Barrat and M. Weigt, cond-mat/9903411. 


\section{FIGURES}

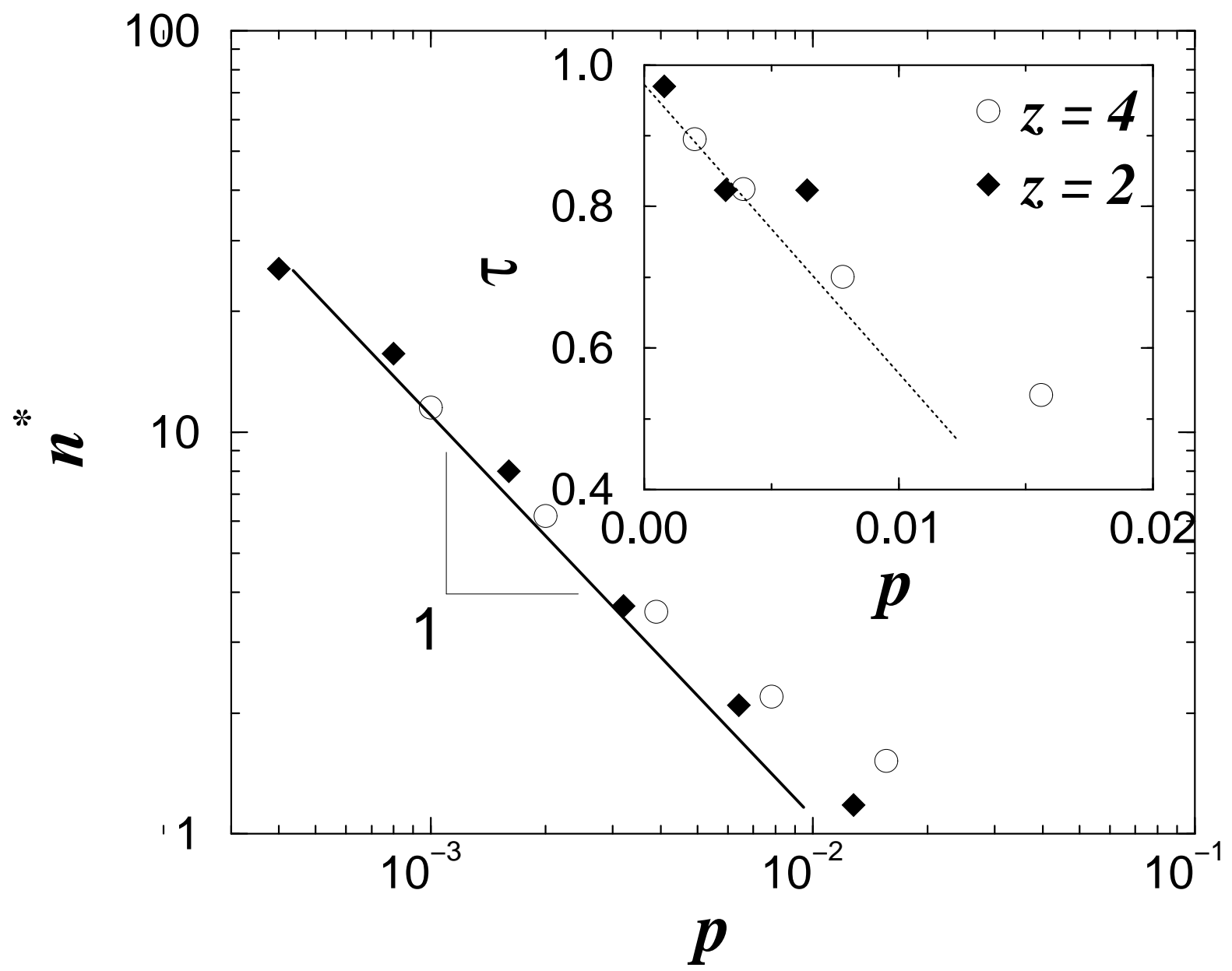

FIG. 1. Loglog plot of $n^{*}$ vs $p$ for system sizes up to 5500 and for $z=2$, 4. Note that the curvature of $n^{*}(p)$ in the $\log \log$ plot, which gives us a local estimate of $\tau$, is increasing as $p$ decreases. In the inset, we show that $\tau$ approaches 1 as $p \rightarrow 0$. Our new estimate of $\tau$ is $0.97 \pm 0.05$, consistent with the value 1 given by a simple scaling argument. 